\title{
MALNUTRITION PROLONGS THE HOSPITALIZATION OF PATIENTS WITH COVID-19 INFECTION: A CLINICAL EPIDEMIOLOGICAL ANALYSIS
}

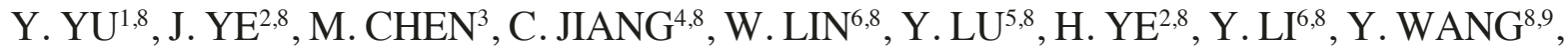 \\ Q. $\mathrm{LIAO}^{8,10}$, D. ZHANG ${ }^{1}$, D. $\mathrm{LI}^{7,8}$ \\ 1. Department of Geriatric, No. 900 hospital of the Joint Logistics Support Force of the Chinese People's Liberation Army, Fujian Medical University, Fuzhou 350025, Fujian, China; \\ 2. Department of Respiratory and Critical Care Medicine, No. 900 hospital of the Joint Logistics Support Force of the Chinese People's Liberation Army, Fuzhou 350025, Fujian, \\ China; 3. Department of Cardiology, No. 900 hospital of the Joint Logistics Support Force of the Chinese People's Liberation Army, Fuzhou 350025, Fujian, China; 4. Department of \\ Gastroenterology, No. 900 hospital of the Joint Logistics Support Force of the Chinese People's Liberation Army, Fuzhou 350025, Fujian, China; 5. Department of Infection, No. 900 \\ hospital of the Joint Logistics Support Force of the Chinese People's Liberation Army, Fuzhou 350025, Fujian, China; 6. Department of Cardiothoracic Surgery, No. 900 hospital of the

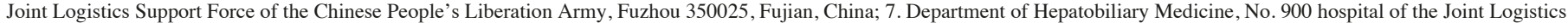

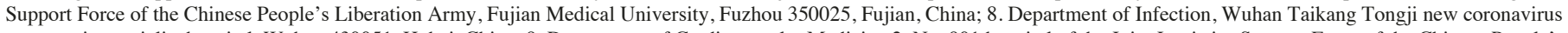

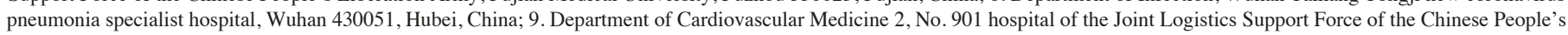 \\ Liberation Army, Hefei 230031, Anhui, China; 10. Department of Oncology, No. 907 hospital of the Joint Logistics Support Force of the Chinese People's Liberation Army, Nanping

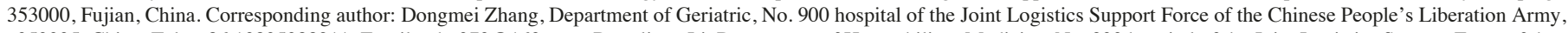 \\ 350025, China, Tel.: +86 13805088911; Email: zdm073@163.com; Dongliang Li, Department of Hepatobiliary Medicine, No. 900 hospital of the Joint Logistics Support Force of the \\ Chinese People's Liberation Army, 350025, China, Tel.: +86 13665052006, Email: dongliangli93@163.com
}

\begin{abstract}
Objectives: During the 2019 coronavirus disease (COVID-19) outbreak, malnutrition may contribute to COVID-19 adverse outcomes. We conducted a clinical epidemiological analysis to investigate the association of malnutrition with hospitalized duration in patients with COVID-19. Design: Retrospective survey study. Setting: Taikang Tongji (Wuhan) hospital in Wuhan, China. Participants: 139 patients with COVID-19. Methods: In total, 139 patients with COVID-19 from patients in the Infection Department of Taikang Tongji (Wuhan) hospital from February 2020 to April 2020 were analyzed retrospectively. We used the "Global leadership Initiative on Malnutrition(GLIM)" assessment standard published in 2019 to assess nutritional status. Prolonged hospitalization was lasting more than the median value of the hospitalized days (17 days) in this population. Results: According to the assessment results of GLIM nutrition assessment, the patients were divided into malnutrition group and normal nutrition group. Compared with the patients in the normal nutrition group, the hospitalization time was longer(15.67 \pm 6.26 days versus $27.48 \pm 5.04$ days, $\mathrm{P}=0.001)$. Kaplan-Meier analysis showed patients with malnutrition were more likely to be hospitalized longer compared with those normal nutrition (mean with 95\% confidence interval [CI]: 28.91[27.52-30.30] versus 22.78[21.76-23.79], P $=0.001$ ). COX regression analysis showed that malnutrition (hazard ratio $[\mathrm{HR}]=3.773$, $\mathrm{P}$ for trend $=0.001$ ) was proportional associated with being discharged from hospital delayed. Conclusion and implications: Present findings suggested that malnutrition contributed to predicting a probability of prolonged hospitalization in patients with COVID-19 infection, to whom extra attentions and precautions should be paid during clinical treatments. Based on the existing results, it is recommended that inpatients with nutritional risk or malnutrition start nutritional support treatment as soon as possible.
\end{abstract}

Keywords: Coronavirus, lymphocyte, nutritional situation, inflammation.

\section{Introduction}

In December 2019, a series of acute respiratory diseases caused by severe acute respiratory syndrome coronavirus 2(SARS-CoV-2) infection occurred in Wuhan, China. It was officially named 2019 coronavirus disease (COVID-19) by the World Health Organization on February 11, 2020 (1). SARSCoV-2 and SARS-CoV, Middle East respiratory syndrome coronavirus (MERS-CoV) belong to the same coronavirus, with typical characteristics of the coronavirus family (2-4). Nutritional status of Covid-19 hospitalized adults has not been fully investigated. So far, only one study in Wuhan, China, is related to the nutritional status of COVID-19 patients (5). This study found that $27.5 \%$ of patients over 65 years old were at risk of malnutrition and $52.7 \%$ were malnourished. Previous studies suggest that malnutrition has been associated with longer lengths of stay in hospital $(6,7)$. The influence of malnutrition on the disease course and recovery of COVID-
19, which remained to be unclear, is of importance to investigate. Therefore, the present study conducted a clinical epidemiological analysis to investigate the association of malnutrition with hospitalized duration to provide clinical evidence for prevention and treatment of COVID-19.

\section{Methods}

\section{Patients}

Novel Coronary Pneumonia(NCP) is defined as pneumonia caused by SARS-CoV-2. The diagnostic criteria for NCP are in line with the "Diagnosis and treatment of pneumonia with a new type of coronavirus infection(trial version 7)" published by the National Health Council on March 3, 2020. The subjects were 139 NCP hospitalized patients in the Infection Department of Taikang Tongji (Wuhan) hospital from February 17, 2020, to April 6, 2020. The classification and nutritional status of NCP in each patient were reviewed by 


\section{MALNUTRITION PROLONGS THE HOSPITALIZATION OF PATIENTS WITH COVID-19 INFECTION}

2 senior attending physicians. All patients were conscious and willing to be assessed and signed informed consent. Exclusion criteria (anyone satisfied): patients with severe disturbance of consciousness and confusion, patients who refused assessment, and patients with severe heart, liver, kidney, and other organ failures, tuberculosis, tumor.

\section{Definition of malnutrition}

We used the "Global leadership Initiative on Malnutrition(GLIM)" assessment standard published in 2019 to assess nutritional status (8), which proposes a more comprehensive assessment procedure for malnutrition three steps based on GLIM criteria. The first step is nutritional screening. Nutritional risk screening 2002(NRS2002) was selected as a tool for nutrition screening with a score $\geq$ three considered to be at nutritional risk (9). The second step is malnutrition assessment (diagnosis). It is a step-bystep assessment of patients at risk of screening, which must meet at least one of the following performance and etiological indicators.

Performance measures included: 1 . Involuntary weight loss: weight loss within 6 months $>5 \%$, or after 6 months $>10 \%$. 2 . Body mass index (BMI): $<18.5 \mathrm{~kg} / \mathrm{m} 2(<70$ years) or $<20 \mathrm{~kg} /$ $\mathrm{m}^{2}(>70$ years); 3 . Muscle mass loss: this study recommended according to the GLIM criteria, referring to the Japanese criteria for muscle loss, using the test of human components around the calf, taking male $\leq 30 \mathrm{~cm}$, female calf $\leq 29 \mathrm{~cm}$ as the standard (10).

Etiological indicators included: 1. Altered energy intake: reduced energy demand $\leq 50 \%$ ( $>1$ weeks), or any proportion of decreased energy ( $>2$ weeks), or chronic gastrointestinal symptoms that cause insufficient or dysabsorption in patients; 2 . Inflammatory responses associated with acute disease/ injury or chronic disease. GLIM criteria define an acute disease/injuryrelated inflammatory response as follows: severe inflammation may be associated with major infections, burns, trauma or closed head injury; other acute disease/injury-related disorders may be associated with mild to moderate inflammation.

All patients with NCP in this study had acute diseaserelated inflammatory responses that met the GLIM criteria.3. Assessment of the degree of malnutrition: according to the degree of phenotype indicators stratified, divided into moderate malnutrition and severe malnutrition.

\section{Criteria of hospital discharge}

The criteria of hospital discharge were as followings (11):(1) body temperature was kept normal for more than 3 consecutive days; (2) significant reduction of respiratory symptoms; (3) substantial improvement determined by conventional chest radiography detection; (4) at least two consecutively negative results of RT-PCR testing separated by an interval of $\geq 24 \mathrm{~h}$. Prolonged hospitalization was defined as lasting more than the median value of the hospitalized days (17 days) in this opulation (discharging from hospital in time as $\leq 17$ days) (11).

\section{Data collection and analyses}

Data were collected and analyzed retrospectively. The following information was reviewed from patient medical records: demographic, clinical, laboratory, and radiological data. Demographics are represented by age, gender, nationality, permanent residence, etc. Clinical datas included: course of the disease, whether fever, whether gastrointestinal symptoms, and the diagnosis of chronic diseases, namely hypertension, diabetes, coronary heart disease, chronic obstructive pulmonary disease (COPD) and other basic diseases. Laboratory indicators were collected after admission, including blood routine, biochemistry, $\mathrm{C}$ reactive protein (CRP), procalcitonin (PCT), interleukin 6 (IL-6), troponin $\mathrm{T}$, brain natriuretic peptide precursor (pro-BNP), immunoglobulin. Radiological data was represented by the number of lobes with inflammation in chest CT image.

\section{Statistical analyses}

All data were analyzed using SPSS 21.0 software. The measurement data were expressed by $(\chi \pm s)$, the count data by t-test. Kaplan-Meier curves reflected and compared the hospitalized duration and possibility to be discharged from hospital in time between different groups. Cox regression analysis identified the independent factors associated with being discharged from hospital in time. All reported $\mathrm{P}$ values were two-tailed, and $\mathrm{P}<0.05$ was considered statistically significant.

\section{Results}

In total, 139 patients admitted to our hospital due to NCP from February 2020 to April 2020 were analyzed retrospectively. Of these patients, 72 were male $(51.80 \%)$, and 67 were female $(47.20 \%)$. The oldest patients were 92 year and the youngest 25 year. The average age is $61.47 \pm 14.76$ years. Other details are given in Table 1 .

Table 1

A general survey of hospitalized patients with NCP $(n=139)$

\begin{tabular}{lccc}
\hline Classify & Group & $\begin{array}{c}\text { Number } \\
\text { of cases }\end{array}$ & Composition (\%) \\
\hline Sex & Male & 72 & 51.80 \\
& Female & 67 & 48.20 \\
Age group & $<65$ years & 81 & 58.30 \\
& $\geq 65$ years & 58 & 41.70 \\
History of hypertension & No & 80 & 57.55 \\
History of diabetes & Yes & 59 & 42.45 \\
& No & 105 & 75.54 \\
History of coronary heart disease & Yes & 34 & 24.46 \\
& No & $119 \bullet$ & 85.61 \\
History of chronic bronchitis & Yes & 20 & 14.39 \\
& No & 130 & 93.53 \\
Combined with underlying diseases & Yes & 9 & 6.47 \\
& No & 39 & 28.06 \\
& Yes & 100 & 71.94 \\
\hline
\end{tabular}


Table 2

Clinical analysis of no malnutrition and malnutrition groups in patients with NCP

\begin{tabular}{|c|c|c|c|c|c|}
\hline Variable & Group & No malnutrition & Malnutrition & Chi-square/ $t / Z$ value & P value \\
\hline \multirow[t]{2}{*}{ Age } & $<65$ years & $71(62.3 \%)$ & $10(40.0 \%)$ & 4.186 & 0.041 \\
\hline & $\geq 65$ years & $43(37.7 \%)$ & $15(60.0 \%)$ & & \\
\hline \multirow[t]{3}{*}{ Sex } & Male & $62(54.4 \%)$ & $10(40.0 \%)$ & 1.700 & 0.192 \\
\hline & Female & $52(45.6 \%)$ & $15(60.0 \%)$ & & \\
\hline & $>93 \%$ & $98(86.0 \%)$ & $22(88.0 \%)$ & & \\
\hline \multirow[t]{2}{*}{ History of Hypertension } & No & $62(54.4 \%)$ & $18(72.0 \%)$ & 2.604 & 0.107 \\
\hline & Yes & $52(45.6 \%)$ & $7(28.0 \%)$ & & \\
\hline \multirow[t]{2}{*}{ History of Diabetes } & No & $91(79.8 \%)$ & $14(56.0 \%)$ & 6.299 & 0.012 \\
\hline & Yes & $23(20.2 \%)$ & $11(44.0 \%)$ & & \\
\hline \multirow[t]{2}{*}{ History of Coronary Heart Disease } & No & $95(83.3 \%)$ & $24(96.0 \%)$ & 1.741 & 0.187 \\
\hline & Yes & $19(16.7 \%)$ & $1(4.0 \%)$ & & \\
\hline \multirow[t]{2}{*}{ History of Chronic Bronchitis } & No & $108(94.7 \%)$ & $22(88.0 \%)$ & 0.626 & 0.429 \\
\hline & Yes & $6(5.3 \%)$ & $3(12.0 \%)$ & & \\
\hline \multirow[t]{2}{*}{ CRP } & $\leq 10 \mathrm{mg} / \mathrm{L}$ & $106(93.0 \%)$ & $20(80.0 \%)$ & 2.689 & 0.101 \\
\hline & $>10 \mathrm{mg} / \mathrm{L}$ & $8(7.0 \%)$ & $5(20.0 \%)$ & & \\
\hline \multirow[t]{2}{*}{ PCT } & $\leq 0.05 \mathrm{ng} / \mathrm{ml}$ & $58(50.9 \%)$ & $15(60.0 \%)$ & 0.684 & 0.408 \\
\hline & $>0.05 \mathrm{ng} / \mathrm{ml}$ & $56(49.1 \%)$ & $10(40.0 \%)$ & & \\
\hline \multirow[t]{2}{*}{ IL-6 } & $\leq 7 \mathrm{pg} / \mathrm{ml}$ & $91(79.8 \%)$ & $18(72.0 \%)$ & 0.742 & 0.389 \\
\hline & $>7 \mathrm{pg} / \mathrm{ml}$ & $23(20.2 \%)$ & $7(28.0 \%)$ & & \\
\hline \multirow[t]{2}{*}{ WBC } & $\leq 3.49 \times 10^{\wedge} 9 / \mathrm{L}$ & $4(3.5 \%)$ & $4(16.0 \%)$ & 3.820 & 0.051 \\
\hline & $>3.5 \times 10^{\wedge} 9 / \mathrm{L}$ & $110(96.5 \%)$ & $21(84.0 \%)$ & & \\
\hline \multirow[t]{2}{*}{$\mathrm{LC}$} & $<1.1 \times 10^{\wedge} 9 / \mathrm{L}$ & $12(10.5 \%)$ & $7(28.0 \%)$ & 3.928 & 0.047 \\
\hline & $>1.1 \times 10^{\wedge} 9 / \mathrm{L}$ & $102(89.5 \%)$ & $18(72.0 \%)$ & & \\
\hline NLR & & $1.79(1.16,2.26)$ & $2.46(1.79,5.00)$ & -3.533 & 0.001 \\
\hline Hospitalization time & & $15.67 \pm 6.26$ & $27.48 \pm 5.04$ & 8.820 & 0.001 \\
\hline
\end{tabular}

CRP: c-reactive protein; PCT: procalcitonin; IL-6: interleukin-6; WBC: white blood cell; LC: lymphocyte count; NLR: granulocyte/lymphocyte ratio.

\section{Nutritional situation}

According to the NRS2002 score, 75 patients had nutritional risk $(53.96 \%)$. Malnutrition assessment considered the ultimate malnutrition in 25/139(17.98\%) of patients. Of these, Nononautonomous weight loss occurred in 22/139(15.83\%). Adults more than 70 years old with less than BMI $20 \mathrm{~kg} / \mathrm{m} 2$ occurred in $7 / 139(5.03 \%)$ and adults less than 70 years old with less than BMI $18.5 \mathrm{~kg} / \mathrm{m} 2$ occurred in 9/139 (6.57\%). Results of further assessment of the degree of malnutrition, 9 patients exhibited moderate malnutrition $(6.57 \%)$, and 13 patients exhibited severe malnutrition $(9.35 \%)$.

\section{Analysis of clinical features}

According to the assessment results of GLIM nutrition assessment, the patients were divided into no malnutrition group and malnutrition group. Table 2 shows the clinical features of the no malnutrition group and malnutrition group in NCP. In the two groups, there was no significant difference in sex composition, fever, and the vital signs of admission Compared with the patients in the no malnutrition group, the proportion of the elderly in the malnutrition group was high, the proportion of diabetes history was higher $(20.2 \%$ versus $44 \%, \mathrm{p}=0.012$ ) (Fig. 1A), the proportion of low lymphocytes was higher $(10.5 \%$ versus $28.0 \%, p=0.047)$ (Fig. 1B), the neutrophil/lymphocyte ratio (NLR) was higher (1.79[1.16, 2.26] versus $2.46[1.79,5.00], \mathrm{p}=0.001$ ) (Fig. 1C), and the hospitalization time was longer(15.67 \pm 6.26 versus $27.48 \pm 5.04$, $\mathrm{p}=0.001)($ Fig. 1D).

\section{Kaplan-Meier analyses of hospitalized duration}

Kaplan-Meier analysis showed patients with malnutrition were more likely to be hospitalized longer compared with those no malnutrition (mean with $95 \%$ confidence interval [CI]: 28.91[27.52-30.30] versus 22.78[21.76-23.79], $\mathrm{P}=0.001$ ) (Fig. 2). 
Figure 1

Clinical analysis of no malnutrition and malnutrition groups in patients with NCP

A. Comparison of history of diabetes between no malnutrition and malnutrition groups; B. Comparison of lymphocyte count between no malnutrition and malnutrition groups; C. Comparison of granulocyte/lymphocyte ratio between no malnutrition and malnutrition groups; D. Comparison of hospitalization time between no malnutrition and malnutrition groups

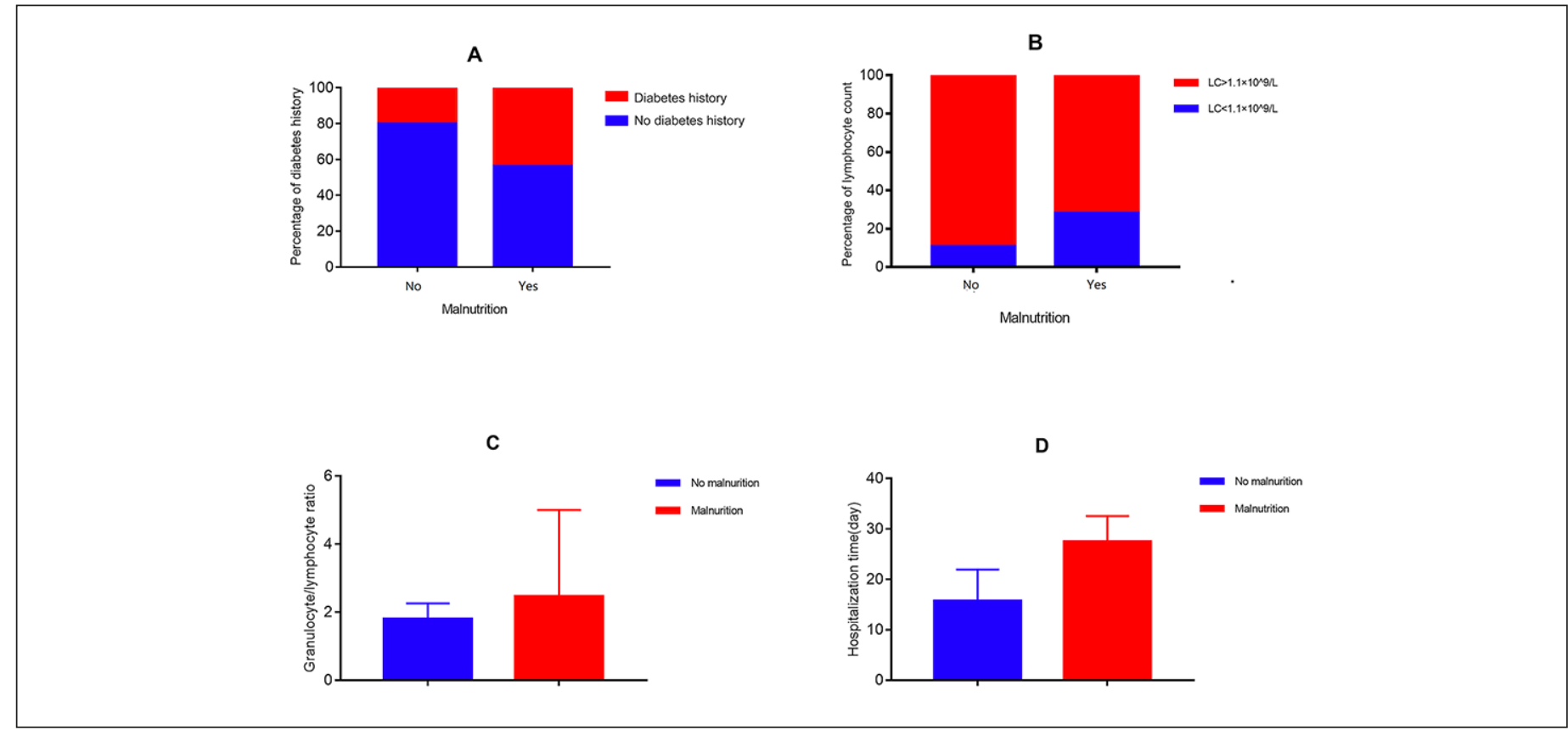

Table 3

COX regression analyses of hospitalization

\begin{tabular}{lcc}
\hline Variables & HR & $\mathbf{9 5 . 0 \%}$ CI \\
\hline Age & 1.167 & $0.588-2.315$ \\
Sex & 1.009 & $0.513-1.984$ \\
History of Hypertension & 0.889 & $0.426-1.856$ \\
History of Diabetes & 1.448 & $0.691-3.038$ \\
History of Coronary Heart Disease & 1.496 & $0.584-3.834$ \\
History of Chronic Bronchitis & 0.802 & $0.228-2.818$ \\
CRP & 0.622 & $0.229-1.691$ \\
PCT & 1.469 & $0.746-2.892$ \\
IL6 & 0.511 & $0.196-1.333$ \\
Troponin T & 1.331 & $0.648-2.734$ \\
WBC count & 1.176 & $0.390-3.545$ \\
Lymphocyte count & 1.908 & $0.605-6.020$ \\
GFR & 0.531 & $0.109-2.585$ \\
NEUTLYM & 0.968 & $0.752-1.247$ \\
Malnutrition & 8.064 & $2.941-22.111$ \\
\hline
\end{tabular}

\section{COX regression analyses of hospitalization}

Defining being discharged from hospital delayed as outcome, COX regression analysis showed that malnutrition (hazard ratio $[\mathrm{HR}]=3.773, \mathrm{P}$ for trend $=0.001$ ) was proportional associated with being discharged from hospital delayed. After adjustment of age, gender, WBC count, lymphocyte count, CRP, PCT, IL-6, troponin, GFR, NEUTLYM, History of hypertension, diabetes, coronary heart disease, chronic bronchitis, malnutrition $(\mathrm{HR}=7.761, \mathrm{P}$ for trend $=0.001$ ) remained to be independently and proportional associated with being discharged from hospital delayed (Table 3).

\section{Discussion}

The present study discovered that malnutrition predisposed patients with COVID-19 to prolonged hospitalization. This is similar in other diseases, consistent with studies such as ZHENG (12). Malnutrition were independently and inversely associated with being discharged from hospital in time for these patients. Because malnutrition patients have poor resistance to disease and slow absorption of inflammation, hospitalization is longer than normal nutrition patients.

Among NCP patients, malnourished patients are older, which may be a cause of long hospital stay. According to the current research in China (13), all age groups have no resistance to the new coronavirus, so long as the transmission conditions can be met. Nearly half of the patients were overaged 50 years (47.7\%) (13). The elderly and people with basic diseases are more severe after infection (14). Because of the complex condition of elderly patients, and relatively long treatment period, there is a high proportion of nutritional risk and malnutrition (15). Older patients are more prone to malnutrition. The proportion of elderly patients with NCP complicated with malnutrition was significantly higher than that of normal nutrition group. 


\section{THE JOURNAL OF NUTRITION, HEALTH \& AGING}

Figure 2

Kaplan-Meier analyses of hospitalized duration

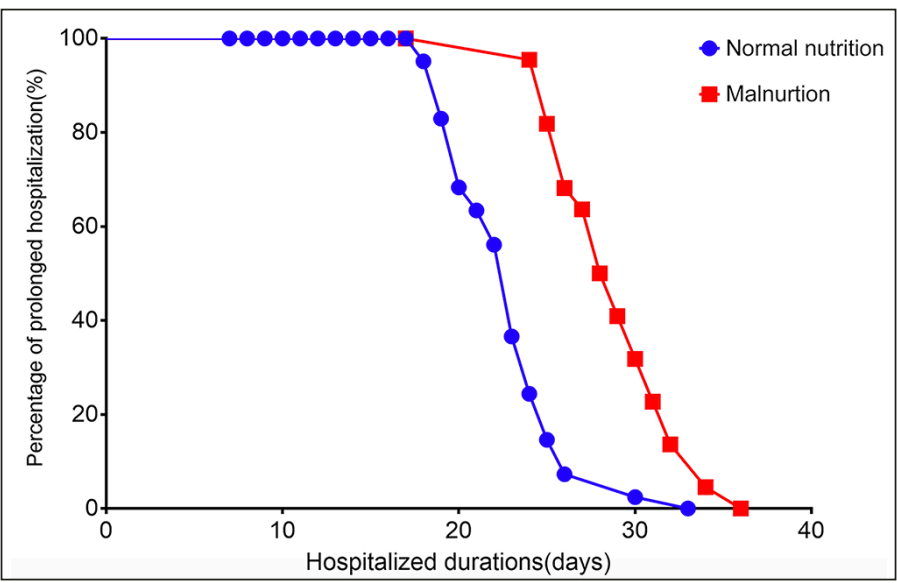

NCP patients with malnutrition have more complications, especially chronic diseases and digestive system symptoms, which may be a cause of long hospital stay. Some studies have shown that patients with chronic basic history generally have a long course of the disease, serious condition, rapid progress, and poor prognosis (16). The more types of chronic diseases combined, the worse the nutritional status of patients with new coronavirus pneumonia (17). In this study, $71.94 \%$ of patients had a history of complicated chronic diseases.

Currently, there is still a lack of targeted methods to identify an effective treatment for COVID-19. Improving immunity, symptomatic treatment, and supportive therapy are the main measures at present. With the prevalence of NCP, the elderly with poor basic nutritional status, and patients with chronic diseases are more critical after infection, highlighting the importance of nutritional treatment (18). Nutritional treatment is a basic treatment method and one of the core contents of comprehensive treatment measures for patients with NCP (18). Previous studies suggest that nutritional support therapy improves clinical outcomes in patients with nutritional risk (19, 20). Through nutrition risk screening and assessment, early detection of patients in need of nutrition intervention is of great significance in the comprehensive treatment of new coronary pneumonia.

\section{Conclusions and implications}

In conclusion, malnutrition contributed to prolonged hospitalization. With high risk of COVID-19, individuals with malnutrition required close monitoring and prevention. Based on the existing results, it is recommended that inpatients with nutritional risk or malnutrition start nutritional support treatment as soon as possible.

Declarations of interest: The authors declare that they have no known competing financial interests or personal relationships that could have appeared to influence the work reported in this paper.

Author's Contributions: Yuhua Yu, Dongmei Zhang and Dongliang Li had full access to all of the data in the study and take responsibility for the integrity of the data and the accuracy of the data analysis. Yuhua Yu, Meixian Chen and Jia Ye contributed equally and share first authorship. Dongmei Zhang and Dongliang Li contributed equally to this article. All authors read and approved the final manuscript.

Source of funding: The study was supported by Taikang Tongji (Wuhan) Hospital 2020 Research Project (No.: TKTJKY2020051) and No. 900 hospital of the Joint Logistics Support Force of the Chinese People's Liberation Army project in-hospital (No.: 2016L01).

Ethical standards: Each patient was treated in accordance with the ethical principles outlined in the Declaration of Helsinki, and consent was obtained from each patient for use of their medical records.The following information, including demographic, clinical, laboratory, and radiological data, was collected from hospital records following discharge.

\section{References}

1. Zhao RS, Yang YH, Yang L, et al. New Coronavirus Infection: Expert Consensus on Guidance and Prevention Strategies for Hospital Pharmacists and the Pharmacy Workforce. Chin Pharm J. 2020. 55(4): 268-277.

2. Wu F, Zhao S, Yu B, et al. A new coronavirus associated with human respiratory disease in China. Nature. 2020. 579(7798): 265-269.

3. Zhou P, Yang XL, Wang XG, et al. A pneumonia outbreak associated with a new coronavirus of probable bat origin. Nature. 2020. 579(7798): 270-273.

4. Zhu N, Zhang D, Wang W, et al. A Novel Coronavirus from Patients with Pneumonia in China, 2019. N Engl J Med. 2020. 382(8): 727-733.

5. Li T, Zhang Y, Gong C, et al. Prevalence of malnutrition and analysis of related factors in elderly patients with COVID-19 in Wuhan, China. Eur J Clin Nutr. 2020. 74(6): 871-875.

6. Coats KG, Morgan SL, Bartolucci AA, Weinsier RL. Hospital-associated malnutrition: a reevaluation 12 years later. J Am Diet Assoc. 1993. 93(1): 27-33.

7. Correia MI, Waitzberg DL. The impact of malnutrition on morbidity, mortality, length of hospital stay and costs evaluated through a multivariate model analysis. Clin Nutr. 2003. 22(3): 235-9.

8. Cederholm T, Jensen GL, Correia M, et al. GLIM criteria for the diagnosis of malnutrition - A consensus report from the global clinical nutrition community. J Cachexia Sarcopenia Muscle. 2019. 10(1): 207-217.

9. Kondrup J, Rasmussen HH, Hamberg O, Stanga Z, Ad Hoc ESPEN Working Group Nutritional risk screening (NRS 2002): a new method based on an analysis of controlled clinical trials. Clin Nutr. 2003. 22(3): 321-36.

10. Maeda K, Ishida Y, Nonogaki T, Mori N. Reference body mass index values and the prevalence of malnutrition according to the Global Leadership Initiative on Malnutrition criteria. Clin Nutr. 2020. 39(1): 180-184.

11. Hu X, Pan X, Zhou W, et al. Clinical epidemiological analyses of overweight/obesity and abnormal liver function contributing to prolonged hospitalization in patients infected with COVID-19. Int J Obes (Lond). 2020

12. Zheng H, Huang Y, Shi Y, Chen W, Yu J, Wang X. Nutrition status,nutrition support therapy,and food intake are related to prolonged hospital stays in china:Results from the nut-ritionday 2015 survey. Ann Nutr Metab. 2016. 69(3-4): 215-225.

13. Yang Yang QL, Mingjin Liu YW, Anran Zhang NJ, et al. Epidemiological and clinical features of the 2019 novel coronavirus outbreak in China. medRxiv. 2020 : doi: https:// doi.org/10.1101/2020.02.10.20021675.

14. Yinghui Jin, Lin Cai, Zhenshun Cheng. A rapid advice guideline for the diagnosis and treatment of 2019 novel coronavirus (2019-nCoV) infected pneumonia (standard version). PLA Medical Journal. 2020. 45(1): 1-20.

15. Ding X, Quan H. Advances in the Study of Safety Influencing Factors in Elderly Inpatients. Medicine Journal of Yanbian University. 2017. 40(04): 299-301.

16. Yingjie Zhuang, Zhu Chen, Jin Li. Clinical and epidemiological characteristics of 26 cases of 2019 novel coronavirus pneumonia. Chinese Journal of Hospital Infection. 2020. 30(06): 826-829.

17. Cao J, Wen M, Shi Y, Wu Y, He Q. Study on nutritional status of patients with COVID-19 and its influencing factors. General practice. 2020. 18(9): 1073-1076.

18. Nutrition CSfPaE. Expert Recommendations on Medical Nutrition Therapy for Patients with COVID-19. Chinese Journal of General Surgery (Electronic Edition). 2020. 14(01): 1 .

19. Jie B, Jiang ZM, Nolan MT, et al. Impact of nutritional support on clinical outcome in patients at nutritional risk: a multicenter, prospective cohort study in Baltimore and Beijing teaching hospitals. Nutrition. 2010. 26(11-12): 1088-93.

20. Schuetz P, Fehr R, Baechli V, et al. Individualised nutritional support in medical inpatients at nutritional risk: a randomised clinical trial. Lancet. 2019. 393(10188) 2312-2321. 